\title{
Noninvasive magnetic resonance imaging of the development of individual colon cancer tumors in rat liver
}

\author{
Olaf R.F. Mook1, Ard Jonker¹, Aart C. Strang1, Andor Veltien², Giulio Gambarota², \\ Wilma M. Frederiks¹, Arend Heerschap ${ }^{2}$, and Cornelis J.F. Van Noorden ${ }^{1}$
}

BioTechniques 44:529-535 (April 2008)

doi 10.2144/000112695

\begin{abstract}
Monitoring tumor development is essential for the understanding of mechanisms involved in tumor progression and to determine efficacy of therapy. One of the evolving approaches is longitudinal noninvasive magnetic resonance imaging (MRI) of tumors in experimental models. We applied high-resolution MRI at 7 Tesla to study the development of colon cancer tumors in rat liver. MRI acquisition was triggered to the respiratory cycle to minimize motion artifacts. A special radio frequency $(R F)$ coil was designed to acquire detailed T1-weighted and T2-weighted images of the liver. T2-weighted images identified hyperintense lesions representing tumors with a minimum diameter of $2 \mathrm{~mm}$, enabling the determination of growth rates and morphological aspects of individual tumors. It is concluded that high-resolution MRI using a dedicated RF coil and triggering to the respiratory cycle is an excellent tool for quantitative and morphological analysis of individual diffusely distributed tumors throughout the liver. However, at present, MRI requires expensive equipment and expertise and is a time-consuming methodology. Therefore, it should preferably be used for dedicated applications rather than for high-throughput assessment of total tumor load in animals.
\end{abstract}

\section{INTRODUCTION}

Development of tumors is determined by many factors including interactions between cancer cells and their environment $(1,2)$. These factors play a role in different stages of tumor development, and inhibition of these processes to hamper tumor growth may have its effects in specific stages only (3). For a proper understanding of tumor development and the effects of intervention studies, longitudinal analysis of tumor development would be of great help (4).

Subcutaneously implanted tumors can be analyzed rather easily in a quantitative manner in time using calipers $(5,6)$, but these tumors do not develop in their natural environment. To mimic tumor development and metastasis in humans as closely as possible, orthotopic tumor growth is preferred (7-9). A number of noninvasive imaging modalities are available to longitudinally monitor tumor development in small animals such as bioluminescence imaging (BLI), fluorescence imaging (FLI), magnetic resonance imaging (MRI), computed tomography (CT), ultrasonography (US), single photon emission CT (SPECT), and positron emission tomography (PET) (4,10-12).

BLI and FLI are the methods of choice to quantitate tumor load in animals, because the methods are reasonably valid when imaging is performed in a standardized manner $(13,14)$. They are rapid, relatively inexpensive, and easy to perform. The spatial resolution of BLI is low, whereas FLI has higher spatial resolution than BLI (14-16). Cancer cells have to be genetically modified for BLI and FLI, because the genes encoding for luciferase, green fluorescent protein (GFP), or related proteins have to be transfected. This may affect the immunogenicity of cancer cells (13). Furthermore, the luminescence and fluorescence signal is quenched by tissues and particularly hair on skin.

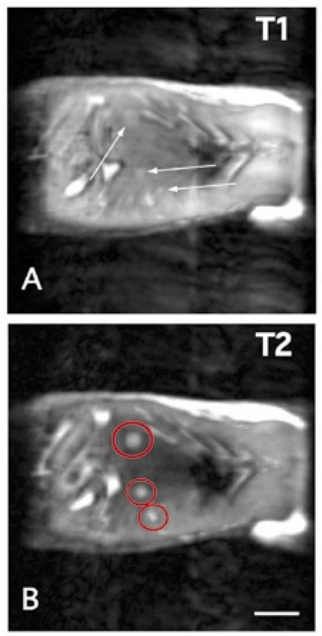

Figure 1. (A) T1-weighted and (B) T2-weighted magnetic resonance (MR) images from the same 2-mm-thick optical section of a rat liver at 5 weeks after induction of colon cancer tumors. Three tumors are faintly visible as hyperintense structures in the T1-weighted image (white arrows), whereas all tumors are clearly visible as hyperintense lesions in the T2-weighted images (encircled in red). Scale bar, $1 \mathrm{~cm}$. 


\section{Research Reports}

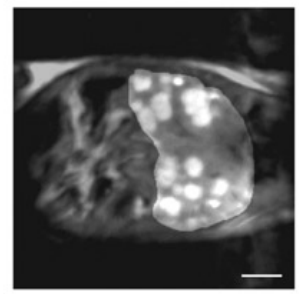

Figure 2. T2-weighted magnetic resonance (MR) image of a 2-mm-thick optical section of the abdomen of a rat. The area of the liver is highlighted to clearly distinguish the individual globular tumors (white structures). The MR image was taken at day 36 after inoculation of colon cancer cells. Scale bar, $1 \mathrm{~cm}$.

Nude or shaven mice are used for BLI and FLI to minimize quenching $(13,17)$. Light has to traverse tissues only once for BLI (light is generated by the luciferase enzyme) and twice for FLI (both excitation and emission light).

MRI has shown its merits for morphological imaging, because it has a high spatial resolution especially in soft tissues, it does not require transfection of cancer cells or radioactivity, and hair on the skin does not interfere with the signal. However, animal MR systems are expensive, expertise is required, and the slow imaging procedures do not allow high-throughput screening (10-12); however, specific approaches are emerging to improve this situation (18). Therefore, MRI has to be used where other imaging techniques have failed.

MRI has proven its value for imaging solitary tumors such as intracranial glioma (19-21) and metastases (22), orthotopic prostate (23) and breast tumors (24), and ectopic subcutaneous tumors $(25,26)$. These tumors can be imaged with the use of long image capturing times because motion artifacts in the living animals do not play a significant role in these locations. MRI has also been applied to quantify tumor load in organs that are subject to movement due to heart beat and/or breathing. These organs include lung (27), colon (28), pancreas $(29,30)$, stomach (31), and liver (32-36).

In the present study, MRI is applied to study quantitatively and morphologically the growth and development of individual diffusely distributed
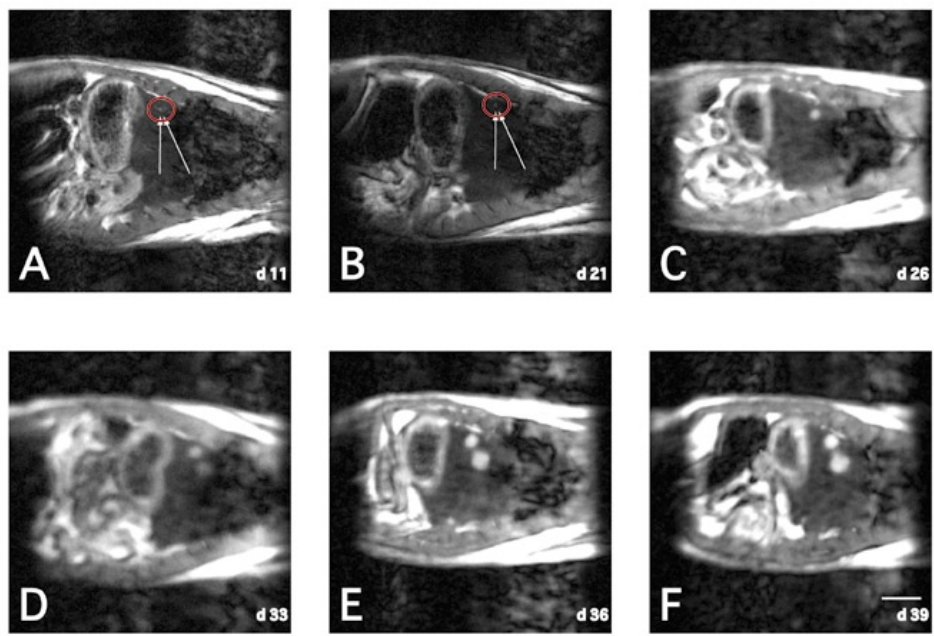

Figure 3. T2-weighted magnetic resonance (MR) images from the same 2-mm-thick optical section of a rat liver. The images were taken at (A) day $11,(\mathrm{~B})$ day $21,(\mathrm{C})$ day 26, (D) day 33, (E) day 36, and (F) day 39 after inoculation of colon cancer cells. Two tumors become visible on day 11 (encircled in red) and collide at day 39 . Scale bar, $1 \mathrm{~cm}$.

colon cancer tumors in rat liver. An optimized technique was used to perform T1- and T2-weighted MRI at 7 Tesla with a dedicated radiofrequency (RF) microstrip coil to increase the signal-to-noise ratio in combination with respiratory triggering to minimize motion artifacts (37).

\section{MATERIALS AND METHODS}

\section{Animals}

Colon cancer tumors were induced as described previously (38) in adult male Wag-Rij rats $(n=26$; Broekman, Someren, The Netherlands) with a body weight of 200-250 g after being maintained for 2 weeks under constant environmental conditions with free access to food and water. All animal experiments were approved by the Animal Care and Use Committees of the Academic Medical Center at the University of Amsterdam and the Nijmegen University Medical Center.

\section{Colon Cancer Cells}

An established colon carcinoma cell line, CC531s, was used. The cells were cultured at $37^{\circ} \mathrm{C}$ as monolayers in RPMI-1640 Dutch modification without L-glutamine (Gibco/BRL,
Grand Island, NY, USA) supplemented with $10 \%$ (v/v) fetal calf serum, $2 \mathrm{mM}$ L-glutamine, $100 \mathrm{IU}$ penicillin $/ \mathrm{mL}$, and $100 \mathrm{mg}$ streptomycin/mL (all from Gibco/BRL). Cells were washed with phosphate-buffered saline (PBS) and detached with trypsin $(0.05 \% \mathrm{w} / \mathrm{v}$; Gibco/BRL) and EDTA $(0.02 \% \mathrm{w} / \mathrm{v}$; Sigma, St. Louis, MO, USA) in PBS. After centrifugation at $250 \times g$ at room temperature for $5 \mathrm{~min}$, cell suspensions were obtained with a viability of at least $95 \%$.

\section{Surgery}

A small midline incision was made in the abdominal wall of rats under anesthesia with fentanyl-fluanizonemidazolam mixture (FFM; $1 \mathrm{~mL}$ Hypnorm, $1 \mathrm{~mL}$ midazolam, and $2 \mathrm{~mL}$ water, $0.27 \mathrm{~mL} / 100 \mathrm{~g}$ body weight, intraperitoneally). A suspension containing 5 $\times 10^{5}$ cancer cells in $500 \mu \mathrm{L}$ PBS was injected into the portal vein with a 27 gauge needle.

\section{Magnetic Resonance Imaging}

MRI of the livers of each rat was performed at various time points during 5 weeks after administration of the cancer cells. A maximum of six animals per day could be imaged. The animals were anesthetized with $1.5 \%$ isoflurane 


\section{Research Reports}

and a mixture of $\mathrm{N}_{2} \mathrm{O} / \mathrm{O}_{2}(1: 1)$. Body temperature was maintained at $37^{\circ} \mathrm{C}$ by covering the rats with a bed heated with circulating warm water. A dedicated $5 \times 7 \mathrm{~cm}$ microstrip RF coil of novel design was used as a transmitter/receiver to improve the quality of the images (37). A customized plastic cradle was built to accommodate the rat in the prone position on the coil.

MR data were collected on a SMIS MR console (Surrey Medical Imaging Systems, Surrey, UK) interfaced with a $7 \mathrm{~T} / 200-\mathrm{mm}$ horizontal bore magnet (Magnex Scientific, Abingdon, UK) and a 150 milliTelsa/meter (mT/m) gradient insert. After initial monitoring of the liver with fast gradient-echo scout images, 16 contiguous coronal images (i.e., parallel to the coil) were acquired. The acquisition protocol consisted of T1- and T2-weighted spin echo images for anatomical localization of the liver and detection of tumors (37). Imaging parameters were: image matrix size of $512 \times 512$, field of view of $14 \times 14 \mathrm{~cm}$, slice thickness of $2 \mathrm{~mm}$, and one signal average per phase-encoding step. The values of repetition time (TR) and echo time (TE) were TR/TE $=500 / 15 \mathrm{~ms}$ for the T1-weighted images and TR/TE $=710 / 36$ $\mathrm{ms}$ for the T2-weighted images. Image acquisition was triggered to the respiration rate monitored by an optical probe (Siracust 401; Siemens, Erlangen, Germany) as described in detail in Gambarota et al. (37).

\section{Data Analysis}

Individual tumors were selected in a specific T2-weighted dataset. Then, the same tumors were identified in $\mathrm{T} 2$-weighted datasets taken at other time points. Individual tumor mass was calculated by two independent methods that gave comparable results. One method was based on the sum of all tumor surface areas $\left(\mathrm{mm}^{2}\right)$ times slice thickness $(\mathrm{mm})$. The second method was based on the image slice with the largest tumor surface area for the calculation of tumor
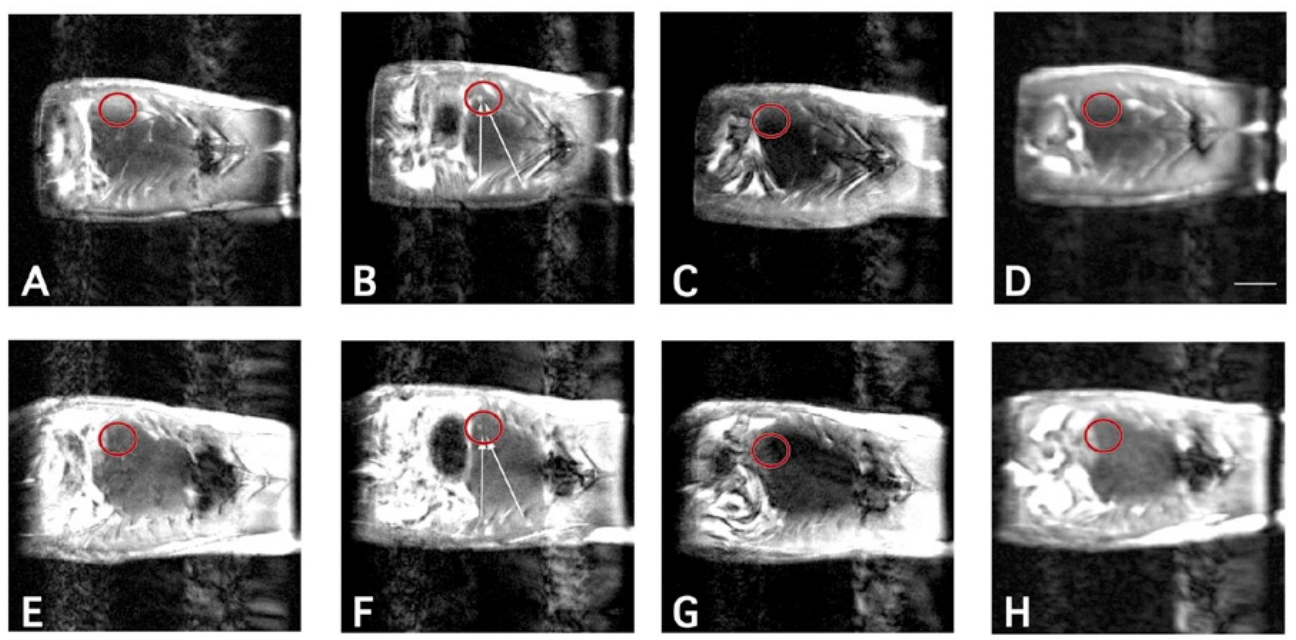

Figure 4. T2-weighted magnetic resonance (MR) images from two adjacent 2-mm-thick optical sections (A, B, C, D and E, F, G, H) of a rat liver. The images were taken at (A and E) day 11, (B and E) day 18, (C and F) day 21, and $(\mathrm{D}$ and $\mathrm{H})$ day 26 after inoculation of colon cancer cells. A tumor becomes visible at day 18 (encircled in red) and has disappeared on day 21 . Scale bar, $1 \mathrm{~cm}$.

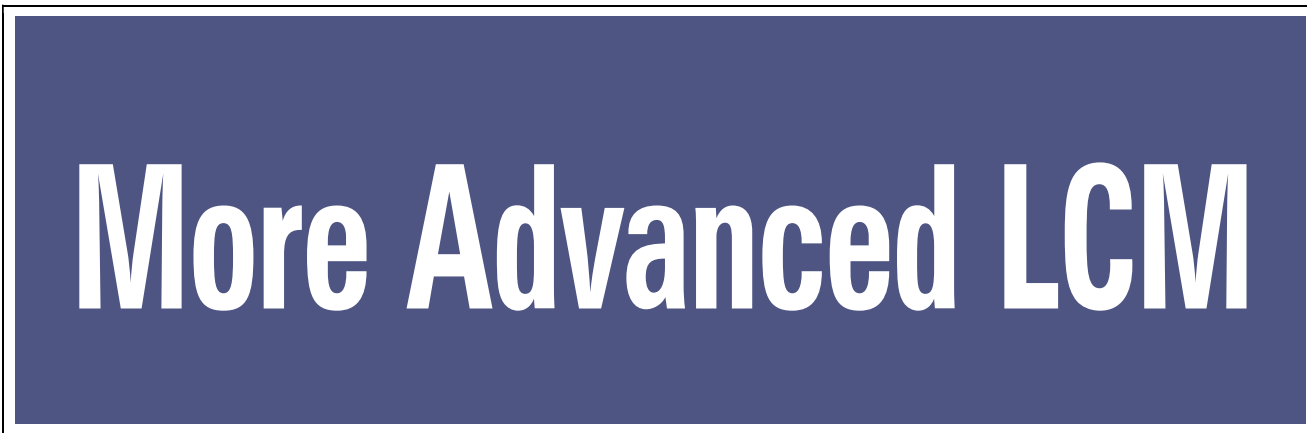

MDS Analytical Technologies introduces the next generation Arcturus $\mathrm{XT}^{\text {'m }}$ microdissection instrument. The Arcturus ${ }^{\mathrm{XT}}$ system takes advantage of the latest advances in microscope technology by employing the all-new Nikon Eclipse Ti-E inverted research microscope as its base.

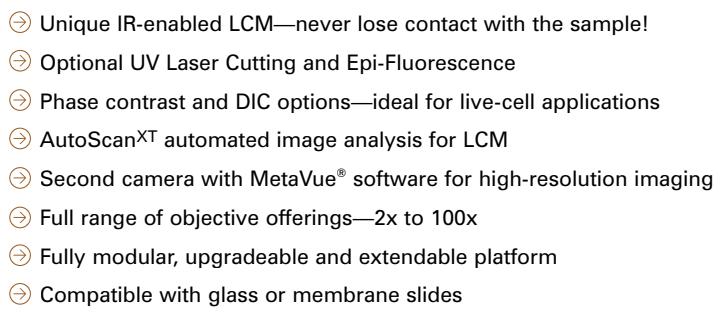

The updated Arcturus ${ }^{\mathrm{XI}}$ system maintains the greatest flexibility in microdissection by combining gentle LCM with rapid UV laser cutting. Together with our Arcturus ${ }^{\circledast}$ microgenomics reagents and Axon GenePix ${ }^{\circledast}$ microarray systems, we provide a more complete solution for microarray research. Visit www.moleculardevices.com/lcm for more information.

Expect more. We'll do our very best to exceed your expectations.

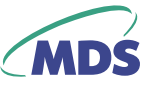

Analytical Technologies

\section{See us at AACR '08} Booth \#1617 


\section{Research Reports}

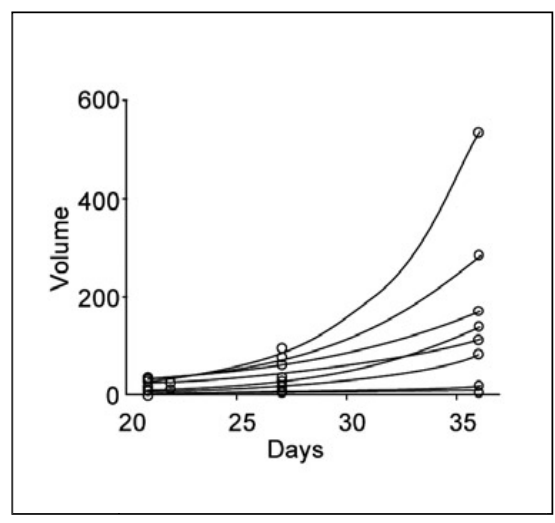

Figure 5. Growth curves of eight individual tumors in livers of rats. Curves are fitted as exponential growth curves.

volumes. As all tumors were more or less globular, the radius was calculated from the largest surface area using $r$ $=\sqrt{ }($ surface area $/ \pi)$. The volume was calculated as $v=4 / 3 \pi r^{3}$. Tumor volume doubling times were calculated on the basis of best fitting exponential curves representing the relationship between volume of individual tumors and time.

\section{RESULTS}

The use of a breathing-triggered MRI protocol enabled sharp and detailed T1-weighted images of the liver and delineation of intrahepatic structures (Figure 1A). T1-weighted imaging did not allow clear visualization of tumors in the liver; some but not all tumors were detected as slightly hyperintense structures (Figure 1A). However, tumors could be clearly identified as hyperintense lesions in T2-weighted images (Figure 1B). A minimum diameter of $2 \mathrm{~mm}$ was required for visualization due to voxel size $(0.27 \times 0.27 \times 2.0 \mathrm{~mm})$. It took approximately 2 weeks before the first tumors could be visualized with MRI. This limitation in spatial resolution is mainly due to the short periods available for imaging during the respiratory cycle of the animals. Fat tissue was also hyperintensive and even more so than tumors in both T1and T2-weighted images (Figure 1). It was discriminated from tumors on the basis of morphology and the fact that it is not present within the liver.

Colon cancer tumors developed as solitary globular tumors even when the liver was full of tumors (Figure 2), but sometimes collision of tumors was observed (Figure 3). Only few tumors disappeared in the period of imaging (3-5 weeks after cancer cell inoculation) (Figure 4).

Imaging of the livers at three or more time points enabled us to follow quantitatively tumor development in time (Figure 5). Exponential fitting of the volumes of individual tumors enabled the calculation of tumor volume doubling time as a measure of tumor growth rate. The average tumor volume doubling time was 0.6 \pm 0.1 week or approximately 4 days in 25 individual tumors in 12 rats. It appeared that the volume doubling time of all individual tumors was similar, whereas their volume varied manifold. This indicated that the starting point of tumor growth varied in time (Figure 6 ), but once the tumors grew, growth rates were similar. Unfortunately, the MRI approach that we used did not allow visualization of tumors in the first 2 weeks after cancer cell inoculation to establish what the fate was of the $5 \times 10^{5}$ inoculated cancer cells that ultimately resulted in only 0-100 tumors per liver (38) or to show different onsets of tumor development.

\section{DISCUSSION}

Studies in the early 1990s have shown that MRI is a powerful tool to quantify tumor load in live animals $(32,34)$ and the effects of therapy on tumor development $(19,33)$. However, MRI of small animals, requiring specialized expensive equipment, technological expertise, and imaging procedures, is slow. In our study, MRI of six animals was the maximum that could be achieved during a working day. Therefore, MRI is not yet the imaging methodology of choice for large-scale determination of tumor load in animals and the effects of therapy thereupon. For these purposes, a simple, rapid, and relatively cheap imaging methodology such as BLI (13) and FLI $(6,14-16)$ is required. In this context, MRI may be more appropriate for the analysis of the development of individual tumors in animals, both morphologically and quantitatively, as shown in the present study. A combi- nation of MRI and BLI (39-42) or MRI and FLI $(43,44)$ provides complementary information.

MRI of tumors does not rely on transfection of cancer cells with any reporter gene, unlike BLI and FLI. Imaging can be performed noninvasively multiple
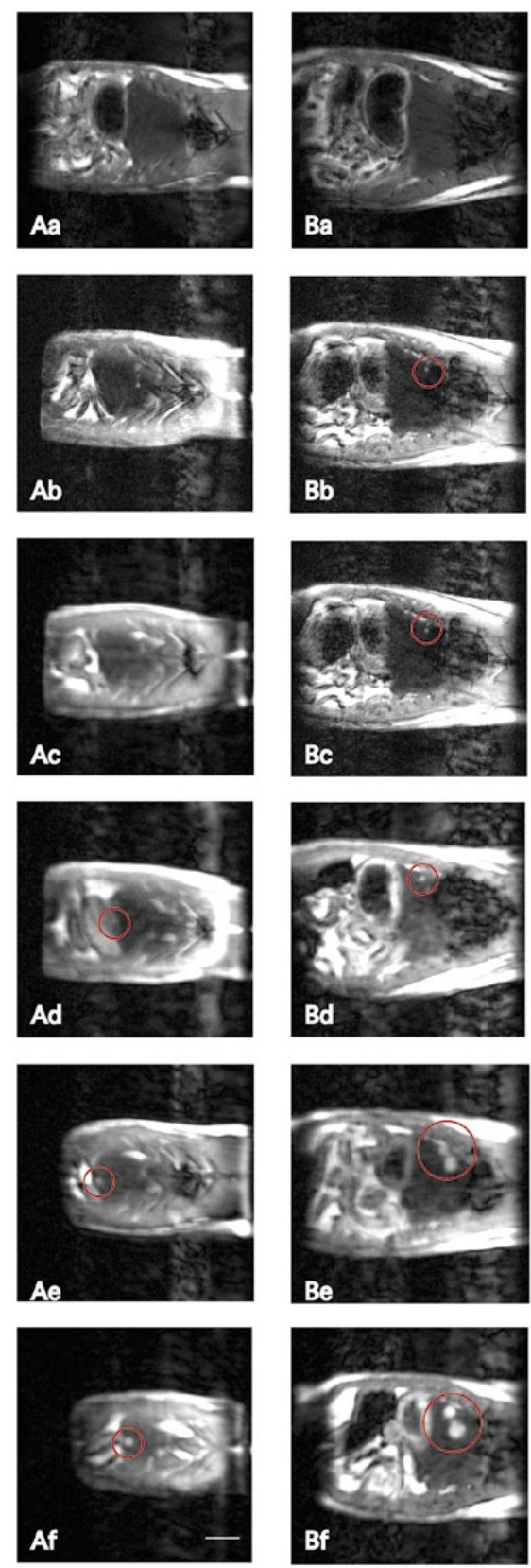

Figure 6. Two series of T2-weighted MR images (A and B) of 2-mm-thick optical sections of a rat abdomen showing the liver. The images were taken at (a) day 11 , (b) day 18 , (c) day 21 , (d) day 26 , (e) day 33, and (f) day 36 after inoculation of cancer cells. Two tumors (encircled in red) develop but become visible on different days (day 26 in panel Ad and day 18 in panel $\mathrm{Bb}$ ), indicating different time points of the onset of growth. Scale bar, $1 \mathrm{~cm}$. 


\section{Research Reports}

times, and tumors can be imaged in any part of the animal. Therefore, MRI provides a valuable tool for imaging tumor development in live animals. MRI technology has improved considerably during the last decade by the implementation of stronger magnetic fields $(23,28,30,37,45)$, modified coils, and the improvement of imaging protocols that has resulted in better signal-to-noise ratios and increased spatial resolution $(37,46,47)$ and higher throughout approaches (18).

The routine procedure used in the present study enabled imaging of tumors with a minimum diameter of $2 \mathrm{~mm}$. MRI of the liver in live animals is difficult due to motion artifacts caused by respiration. Triggering of the MRI protocol to the breathing of the animal enabled us to acquire well-contrasted and detailed images of rat livers and to image tumor development of colon cancer in the liver, as was also shown by Garbow et al. (27) and Cai et al. (36). These improvements in MRI technology enable good quality noninvasive imaging of tumors in living animals.

Quantification of growth of individual tumors appeared to be possible. Tumor volume doubling times obtained by exponential curve fitting of tumor volumes at 3,4 , and 5 weeks showed that the volume doubling time was similar in all tumors. It suggests that tumors have different onsets in growth rather than different growth rates to explain differences in sizes at the time of sacrifice of the animals.

A number of technological modifications of MRI may be of interest to further improve contrast and thus spatial resolution, such as the use of the contrast agents gadolinium $(21,24,28,29)$ and manganese (48) in T1 imaging or iron oxide particles (25) in T2 imaging. Other MRI approaches such as diffusion MRI $(20,49)$, which is sensitive to cellular density, and organization or dynamic contrast-enhanced MRI (26,50,51), which reflects vascular functionality, are powerful approaches for the functional characterization of individual tumors. Recently, MRI of targeted agents is showing great promise $(52,53)$.

Finally, Heyn et al. (54) reported an MRI study at the single-cell level of breast cancer metastasis in mouse brain. Cancer cells were labeled with micron-sized iron oxide particles. This methodology allows the study of early events in the development of metastases to address questions, such as what is the fate of cancer cells when entering the circulation and/or the organ where secondary growth takes place, the onset of growth, or the elimination of a tumor.

In conclusion, this study shows that MRI is a highly sophisticated technique that can be applied to address specific research questions in tumor biology, such as the analysis of the development of individual tumors in time both morphologically and quantitatively.

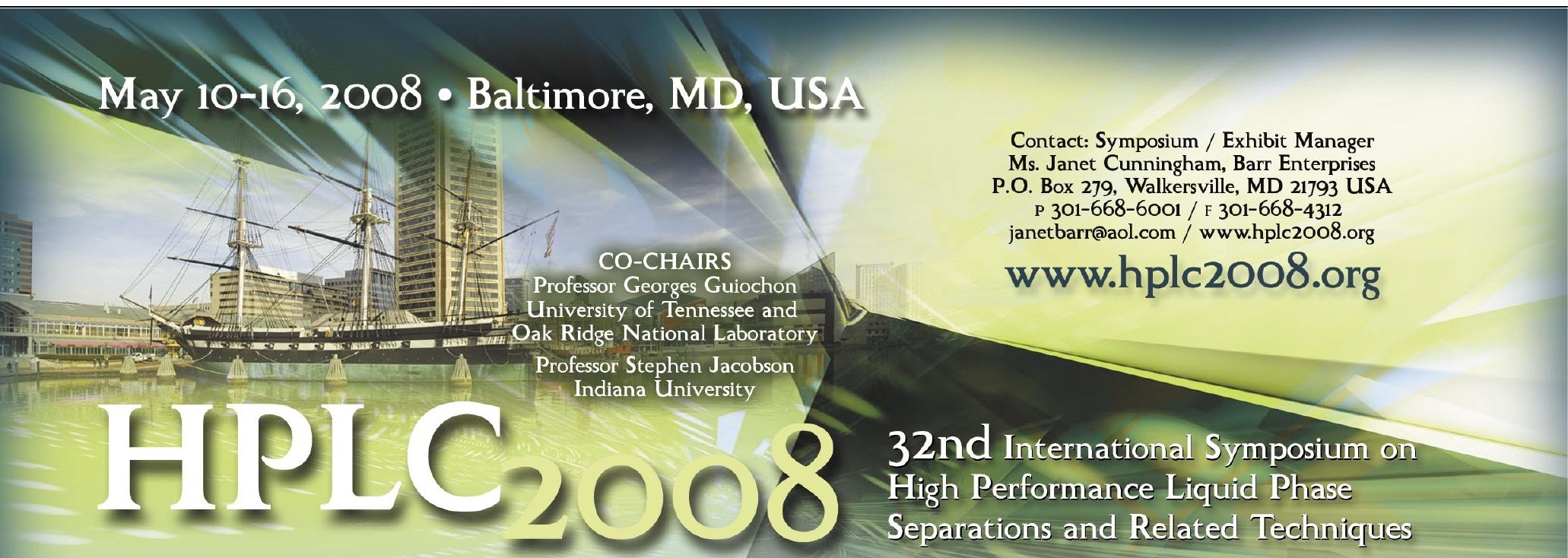

Join scientists from around the world who will focus on presenting practical, novel and cutting-edge areas of scientific research at the largest meeting and exposition in the world dedicated to liquid phase separation science.

The program will address practical and economical needs of the analytical chemist, biochemist, molecular biologist, and those in the separation sciences. Short courses are designed for you to gain insights into the latest, most popular areas of liquid-based separations and apply them to meet your specific needs. Workshops focus on providing quick, effective tools and techniques to address various practical problems. The Exposition is the largest, most comprehensive display of commercial products related to liquid phase separations and analysis.

\section{CALL FOR PAPERS}

Researchers are invited to showcase their contributions to emerging and established topics in the separation sciences by submitting abstracts to HPLC 2008 through our web site at www.hplc2008.org.

\section{TOPIC AREAS}

- Column Technology

- Data acquisition, analysis and handling

- Multi-dimensional Chromatography

- Micro- and Nano-technologies

- Instrumentation

- Sample Preparation

- Theory of Chromatography

- Applications in Life Sciences

- Applications, Other

- Other Separation Methods 


\section{ACKNOWLEDGMENTS}

O.R.F.M. and A.J. contributed equally to this work. The authors thank Mrs. Trees M.S. Pierik for the careful preparation of the manuscript.

\section{COMPETING INTERESTS STATEMENT}

The authors declare no competing interests.

\section{REFERENCES}

1. Van Noorden, C.J.F., L.C. Meade-Tollin, and F.T. Bosman. 1998. Metastasis. Amer. Sci. 86:130-141.

2. Littlepage, L.E., M. Egeblad, and Z. Werb. 2005. Coevolution of cancer and stromal cellular responses. Cancer Cell 7:499-500.

3. Lah, T.T., M.B. Durán Alonso, and C.J.F. Van Noorden. 2006. Antiprotease therapy in cancer: hot or not? Expert Opin. Biol. Ther. 6:257-279.

4. Weissleder, R. 2002. Scaling down imaging: molecular mapping of cancer in mice. Nat. Rev. Cancer 2:11-18.

5. Vassal, G., I. Boland, A. Santos, M.C. Bissery, M.J. Terrier-Lacombe, J. Morizet, C. Sainte-Rose, A. Lellouch-Tubiana, et al. 1997. Potent therapeutic activity of irinotecan (CPT-11) and its schedule dependency in medulloblastoma xenografts in nude mice. Int. J. Cancer 73:156-163.

6. Van Noorden, C.J.F., T.G.N. Jonges, L.C. Meade-Tollin, R.E. Smith, and A. Koehler. 2000. In vivo inhibition of cysteine proteinases delays the onset of growth of human pancreatic cancer explants. Br. J. Cancer 82:931-936.

7. Kubota, T. 1994. Metastatic models of human cancer xenografted in the nude mouse: the importance of orthotopic transplantation. J. Cell. Biochem. 56:4-8.

8. Killion, J.J., R. Radinsky, and I.J. Fidler. 1998. Orthotopic models are necessary to predict therapy of transplantable tumors in mice. Cancer Metastasis Rev. 17:279-284.

9. Hoffman, R.M. 1999. Orthotopic metastatic mouse models for anticancer drug discovery and evaluation: a bridge to the clinic. Invest. New Drugs 17:343-359.

10. Lewis, J.S., S. Achilefu, J.R. Garbow, R. Laforest, and M.J. Welch. 2002. Small animal imaging: current technology and perspectives for oncological imaging. Eur. J. Cancer 38:2173-2188.

11. Lyons, S.K. 2005. Advances in imaging mouse tumour models in vivo. J. Pathol. 205:194-205.

12. Koo, V., P.W. Hamilton, and K. Williamson. 2006. Non-invasive in vivo imaging in small animal research. Cell. Oncol. 28:127-139.

13. Klerk, C.P.W., R.M. Overmeer, T.M. Niers, H.H. Versteeg, D.J. Richel, T. Buckle, C.J. Van Noorden, and O. van Tellingen. 2007. Validity of bioluminescence measurements for noninvasive in vivo imaging of tumor load in small animals. BioTechniques 43:S7-S30.

14. Hoffman, R.M. 2005. The multiple uses of fluorescent proteins to visualize cancer in vivo. Nat. Rev. Cancer 5:796-806.

15. Yang, M., E. Baranov, P. Jiang, F.X. Sun, X.M. Li, L. Li, S. Hasegawa, M. Bouvet, et al. 2000. Whole-body optical imaging of green fluorescent protein-expressing tumors and metastases. Proc. Natl. Acad. Sci. USA 97:1206-1211.

16. Hoffman, R.M. 2004. In vivo imaging with fluorescent proteins: the new cell biology. Acta Histochem. 106:77-87.

17. Schmitt, C.A., J.S. Fridman, M. Yang, S. Lee, E. Baranov, R.M. Hoffman, and S.W. Lowe. 2002. A senescence program controlled by p53 and p16 $16^{\text {INK4a }}$ contributes to the outcome of cancer therapy. Cell 109:335346.

18. Nieman, B.J., N.A. Bock, J. Bishop, X.J. Chen, J.G. Sled, J. Rossant, and R.M. Henkelman. 2005. Magnetic resonance imaging for detection and analysis of mouse phenotypes. NMR Biomed. 18:447-468.

19. Chenevert, T.L., P.E. McKeever, and B.D. Ross. 1997. Monitoring early response of experimental brain tumors to therapy using diffusion magnetic resonance imaging. Clin. Cancer Res. 3:1457-1466.

20. Lee, K.C., D.E. Hall, B.A. Hoff, B.A. Moffat, S. Sharma, T.L. Chenevert, C.R. Meyer, W.R. Leopold, et al. 2006. Dynamic imaging of emerging resistance during cancer therapy. Cancer Res. 66:4687-4692.

21. Jost, S.C., J.E. Wanebo, S.-K. Song, M.R. Chicoine, K.M. Rich, T.A. Woolsey, J.S. Lewis, R.H. Mach, et al. 2007. In vivo imaging in a murine model of glioblastoma. Neurosurgery 60:360-371.

22. Leenders, W., B. Küsters, J. Pikkemaat, P. Wesseling, D. Ruiter, A. Heerschap, J. Barentsz, and R.M. de Waal. 2003. Vascular endothelial growth factor-A determines detectability of experimental melanoma brain metastasis in GD-DTPA-enhanced MRI. Int. J. Cancer 105:437-443.

23. Degrassi, A., M. Russo, E. Scanziani, A. Giusti, R. Ceruti, G. Texido, and E. Pesenti. 2007. Magnetic resonance imaging and histopathological characterization of prostate tumors in TRAMP mice as model for pre-clinical trials. Prostate 67:396-404.

24. Galiè, M., M. D'Onofrio, L. Calderan, E. Nicolato, A. Amici, C. Crescimanno, P. Marzola, and A. Sbarbati. 2004. In vivo mapping of spontaneous mammary tumors in transgenic mice using MRI and ultrasonography. J. Magn. Reson. Imaging 19:570-579.

25. Fan, X., J.N. River, M. Zamora, K. Tarlo, K. Kellar, C. Rinker-Schaeffer, and G.S. Karczmar. 2001. Differentiation of nonmetastatic and metastatic rodent prostate tumors with high spectral and spatial resolution MRI. Magn. Reson. Med. 45:1046-1055.

26. Marzola, P., A. Degrassi, L. Calderan, P. Farace, C. Crescimanno, E. Nicolata, A. Giusti, E. Pesenti, et al. 2004. In vivo assessment of antiangiogenic activity of SU6668 in an experimental colon carcinoma model. Clin. Cancer Res. 10:739-750.
27. Garbow, J.R., Z. Zhang, and M. You. 2004 Detection of primary lung tumors in rodents by magnetic resonance imaging. Cancer Res. 64:2740-2742

28. Hensley, H.H., W. Chang, and M.L. Clapper. 2004. Detection and volume determination of colonic tumors in Min mice by magnetic resonance micro-imaging. Magn Reson. Med. 52:524-529.

29. He, Z., J.L. Evelhoch, R.M. Mohammad, N.V. Adsay, G.R. Pettit, V.K. Vaitkevicius, and F.H. Sarkar. 2000. Magnetic resonance imaging to measure therapeutic response using an orthotopic model of human pancreatic cancer. Pancreas 21:69-76.

30. Grimm, J., A. Potthast, A. Wunder, and A. Moore. 2003. Magnetic resonance imaging of the pancreas and pancreatic tumors in a mouse orthotopic model of human cancer. Int. J. Cancer 106:806-811.

31. Berr, S.S., J.K. Roche, W. El-Rifai, M.F. Smith, Jr., and S.M. Powell. 2003. Magnetic resonance imaging of gastric cancer in Tff1 knock-out mice. Magn. Reson. Med. 49:1033-1036.

32. Qin, Y., M. Van Cauteren, M. Osteaux, and G. Willems. 1992. Quantitative study of the growth of experimental hepatic tumors in rats by using magnetic resonance imaging. Int. J. Cancer 51:665-670.

33. Qin, Y., M. Van Cauteren, M. Osteaux, A.V. Schally, and G. Willems. 1992. Inhibitory effect of somatostatin analogue RC-160 on the growth of hepatic metastases of colon cancer in rats: a study with magnetic resonance imaging. Cancer Res. 52:6025-6030.

34. Mahmood, U., M.L. Devitt, P.G. Kocheril, E. Sutanto-Ward, D. Ballon, E.R Sigurdson, and J.A. Koutcher. 1992 Quantitation of total metastatic tumor volume in the rat liver: correlation of MR and histologic measurements. J. Magn. Reson. Imaging 2:335-340.

35. Trübenbach, J., F. Graepler, P.L. Pereira, P. Ruck, U. Lauer, M. Gregor, C.D. Claussen, and P.E. Huppert. 2000. Growth characteristics and imaging properties of the morris hepatoma 3924A in ACI rats: a suitable model for transarterial chemoembolization. Cardiovasc. Intervent. Radiol. 23:211-217.

36. Cai, S.-R., J.R. Garbow, R. Culverhouse, R.D. Church, W. Zhang, W.D. Shannon, and H.L. McLeod. 2005. A mouse model for developing treatment for secondary liver tumors. Int. J. Oncol. 27:113-120.

37. Gambarota, G., A. Veltien, H. van Laarhoven, M. Philippens, A. Jonker, O.R. Mook, W.M. Frederiks, and A. Heerschap. 2004. Measurements of $T_{1}$ and $T_{2}$ relaxation times of colon cancer metastases in rat liver at 7 T. MAGMA 17:281-287.

38. Griffini, P., O. Fehres, L. Klieverik, I.M.C. Vogels, W. Tigchelaar, S.M. Smorenburg and C.J.F. Van Noorden. 1998. Dietary omega- 3 polyunsaturated fatty acids promote colon carcinoma metastasis in rat liver. Cancer Res. 58:3312-3319.

39. Kemper, E.M., W. Leenders, B. Küsters, S. Lyons, T. Buckle, A. Heerschap, W. Boogerd, J.H. Beijnen, et al. 2006. Development of luciferase tagged brain tumour models in mice for chemotherapy 
intervention studies. Eur. J. Cancer 42:32943303.

40. Rehemtulla, A., L.D. Stegman, S.J. Cardozo, S. Gupta, D.E. Hall, C.H. Contag, and B.D. Ross. 2000. Rapid and quantitative assessment of cancer treatment response using in vivo bioluminescence imaging. Neoplasia 2:491-495.

41. Inoue, Y., K. Izawa, A.T.Y. Nomura, R. Sekine, N. Oyaizu, and K. Ohtomo. 2007. Monitoring of disease progression by bioluminescence imaging and magnetic resonance imaging in an animal model of hematologic malignancy. Exp. Hematol. 35:407-415.

42. Szentirmai, O., C.H. Baker, N. Lin, S. Szucs, M. Takahashi, S. Kiryu, A.L. Kung, R.C. Mulligan, et al. 2006. Noninvasive bioluminescence imaging of luciferase expressing intracranial U87 xenografts: correlation with magnetic resonance imaging determined tumor volume and longitudinal use in assessing tumor growth and antiangiogenic treatment effect. Neurosurgery 58:365-372.

43. Moore, A., Z. Medarova, A. Potthast, and G. Dai. 2004. In vivo targeting of underglycosylated MUC-1 tumor antigen using a multimodal imaging probe. Cancer Res. 64:1821-1827.

44. Bouvet, M., J. Spernyak, M.H. Katz, R.V. Mazurchuk, S. Takimoto, R. Bernacki, Y.M. Rustum, A.R. Moossa, et al. 2005. High correlation of whole-body red fluorescent protein imaging and magnetic resonance imaging on an orthotopic model. Cancer Res. 65:9829-9833.

45. Hogers, B., D. Gross, V. Lehmann, H.J. de Groot, A. de Roos, A.C. Gittenberger-de Groot, and R.E. Poelmann. 2001. Magnetic resonance microscopy at 17.6-Tesla on chicken embryos in vitro. J. Magn. Reson. Imaging 14:83-86.

46. Heerschap, A., M.G. Sommers. H.J. in 't Zandt, W.K. Renema, A.A. Veltien, and D.W. Klomp. 2004. Nuclear magnetic resonance in laboratory animals. Methods Enzymol. 385:41-63.

47. Schmidt, K.F., M. Ziu, N.O. Schmidt, P. Vaghasia, T.G. Cargioli, S. Doshi, M.S. Albert, P.M. Black, et al. 2004. Volume reconstruction techniques improve the correlation between histological and in vivo tumor volume measurements in mouse model of human gliomas. J. Neurooncol. 68:207-215.

48. Chabanova, E., V. Logager, J.M. Moller, H. Dekker, J. Barentsz, and H.S. Thomsen. 2006. Imaging liver metastases with a new oral manganese-based contrast agent. Acad. Radiol. 13:827-832.

49. Ross, B.D., B.A. Moffat, T.S. Lawrence, S.K. Mukherji, S.S. Gebarski, D.J. Quint, T.D. Johnson, L. Junck, et al. 2003. Evaluation of cancer therapy using diffusion magnetic resonance imaging. Mol. Cancer Ther. 2:581-587.

50. Van der Sanden, B.P., T.H. Rozijn, P.F. Rijken, H.P. Peters, A. Heerschap, A.J. Van der Kogel, and W.M. Bovee. 2000. Noninvasive assessment of the functional neovasculature in 9L-glioma growing in rat brain by dynamic $1 \mathrm{H}$ magnetic resonance imaging of gadolinium uptake. J. Cereb. Blood Flow Metab. 20:861-870.
51. Verhoye, M., B.P. Van Der Sanden, P.F. Rijken, H.P. Peters, A.J. Van Der Kogel, G. Pee, G. Vanhoutte, A. Heerschap, et al. 2002. Assessment of the neovascular permeability in glioma xenografts by dynamic $\mathrm{T}(1)$ MRI with Gadomer-17. Magn. Reson. Med. 47:305-313.

52. Allen, M.J. and T.J. Meade. 2004. Magnetic resonance contrast agents for medical and molecular imaging. Met. Ions Biol. Syst. 42:1-38

53. Sosnovik, D.E. and R. Weissleder. 2007. Emerging concepts in molecular MRI. Curr. Opin. Biotechnol. 18:4-10.

54. Heyn, C., J.A. Ronald, S.S. Ramadan, J.A. Snir, A.M. Barry, L.T. Mackenzie, D.J. Mikulis, D. Palmieri, et al. 2006. In vivo MRI of cancer cell fate at the single-cell level in a mouse model of breast cancer metastasis to the brain. Magn. Reson. Med. 56:10011010 .

Received 17 September 2007; accepted 7 November 2007.
Address correspondence to Cornelis J.F. Van Noorden, Department of Cell Biology and Histology, Academic Medical Center, University of Amsterdam, L 3/111, Meibergdreef 15, 1105 AZ Amsterdam, The Netherlands.e-mail: c.j.vannoorden@amc. uva.nl

To purchase reprints of this article, contact: Reprints@BioTechniques.com

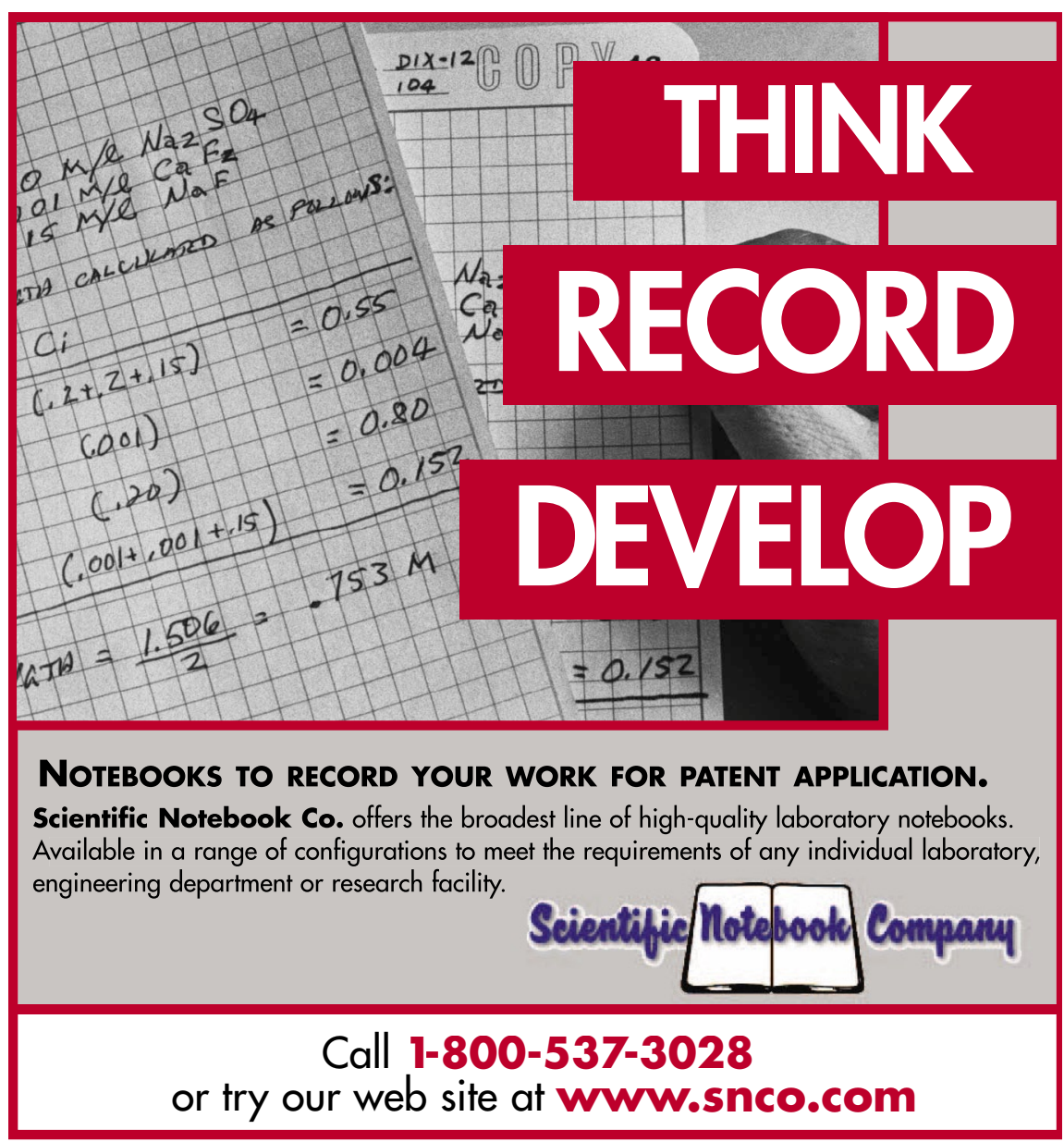

Circle Reader Service No. 172 